\title{
Introduction: Surgery of the Central Skull Base
}

\author{
Vinko V. Dolenc, M.D., Ph.D. \\ Clinical Department of Neurosurgery, Division of Surgery, University Medical Center, Ljubljana, Slovenia
}

$\mathrm{W}$

ITH his anatomical studies of the parasellar space, the so-called cavernous sinus (CS), Taptas opened Pandora's box more than 60 years ago. Parkinson continued the anatomical studies, and operated on vascular lesions in the CS with the help of extracorporeal circulation. The need for endovascular treatment of intracavernous internal carotid artery (ICA) aneurysms, as well as carotid-cavernous fistulas (CCFs), was obvious. Serbinenko started with the endovascular treatment of CCFs and ICA aneurysms using a balloon. At nearly the same time, Hakuba undertook surgical treatment of tumorous lesions in the region. Glascock studied the ICA in relation to the petrous bone, and with his studies of the ICA and this artery's relationship to the other structures, it became clear that further understanding of the pathological entities in the parasellar space hinged on additional microanatomical studies.

At our neurosurgical department, neuroanatomical studies of the CS were systematically conducted, as was actual surgical treatment of vascular and tumorous lesions in patients. In the early 1990 s the direct approach to vascular CS lesions, without extracorporeal circulation, was possible owing to the acquired knowledge of the CS anatomy. In 1986, the first International Symposium on Cavernous Sinus was organized in Ljubljana in response to the increase in treatments of vascular and tumorous lesions in the region. Ever since, the treatment of CS pathological entities has been the subject of heated discussions. The reasons for that have been manifold. Through the years and with the accumulated knowledge of neuroanatomy, surgical treatment has been performed in more and more centers. The endovascular treatment of vascular and some tumorous lesions has become increasingly refined and successful. In addition, Gamma Knife treatment of tumorous lesions has become popular and also successful. Of course, as happens with each new treatment modality, it was mishandled in many cases; however, in combina- tion with microsurgery, Gamma Knife treatment became the gold standard for the remaining tumors as well as for a number of small lesions in the CS amenable to radiosurgery. The same is true for endovascular treatment, which was successfully used for many vascular lesions in the central skull base (not only in the CS), but not for all. In our opinion, a combination of 2 or sometimes also all 3 modalities is needed. It goes without saying that whenever the combination of the modalities is to be applied, agreement among all 3 participant experts should be achieved regarding the order in which each modality should be employed.

25

For the last 2 decades, it has been difficult to exclude the topic of central skull base lesions from the program of any of neurosurgical meeting around the globe. The reports assembled in this issue of Neurosurgical Focus in a way reflect the current status of the practice. It is obvious that diversity of opinion still exists and that universal agreement on a firm protocol is not yet at hand. 